\title{
Fatores inerentes à realização de trabalhos científicos no ACeS de Gondomar: estudo transversal
}

Liliana Teixeira, ${ }^{1}$ Patrícia Fernandes, ${ }^{2}$ Pedro Azevedo, ${ }^{3}$ Sílvia Carvalho ${ }^{4}$

\section{RESUMO}

Objetivos: A investigação é essencial à afirmação e ao desenvolvimento da medicina geral e familiar (MGF) como disciplina científica. Em Portugal têm sido reconhecidas dificuldades à investigação ao nível dos cuidados de saúde primários (CSP). Pretende-se, com o presente estudo, quantificar a produção científica desenvolvida pelos médicos num agrupamento de centro de saúde (ACeS), bem como identificar os fatores inerentes e motivações para a sua realização.

Tipo de estudo: Estudo descritivo, transversal.

Local: ACeS de Gondomar.

População: Médicos de família e internos de formação específica de MGF.

Métodos: Foi realizado um censo de um total de 133 médicos incluídos no estudo, a quem foi entregue um questionário elaborado pelos autores para o efeito. As principais variáveis estudadas foram o número, tipo e forma de divulgação dos trabalhos realizados pelos participantes durante um período de dois anos, bem como a satisfação dos participantes com o número de trabalhos realizados. Foi ainda recolhida informação relativa aos fatores que motivaram a realização dos trabalhos.

Resultados: De um total de 79 questionários obtidos (taxa de resposta global de 59,4\%), foram reportados 212 trabalhos científicos (média de 2,7 trabalhos por médico), sendo que os internos apresentaram um número médio de trabalhos superior ao dos especialistas ( 6 e 1,9, respetivamente) e 46,8\% dos médicos não realizaram qualquer trabalho. A maioria dos participantes (57\%) considerou que o número de trabalhos realizado pelo próprio não foi adequado, sendo que as razões apontadas para tal foram sobretudo a falta de tempo e de horário dedicado à investigação.

Conclusão: A dinâmica da especialidade de MGF está em evolução, assim como os CSP. Há ainda um longo caminho a percorrer no que diz respeito às condições de formação das unidades de saúde, assim como incentivos à realização de investigação de qualidade.

Palavras-chave: Medicina Geral e Familiar; Investigação; Cuidados de Saúde Primários.

\section{INTRODUÇÃO}

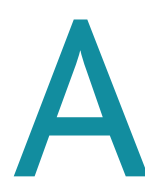
investigação ao nível dos cuidados de saúde primários (CSP) tem sido alvo de intensa discussão. ${ }^{1-3}$ Nos últimos anos muito se evoluiu em Portugal relativamente à construção de competências, sustentabilidade e continuidade, de modo a aumentar a capacidade de desenvolver investigação no Serviço Nacional de Saúde (SNS).

Os médicos de família apresentam um elevado potencial para a realização de estudos científicos de investigação ao nível dos CSP. Pela sua relação estreita com a população apresentam-se numa posição privi- legiada para aceder a doentes representativos da população geral, podendo ainda fazer o seu acompanhamento de forma longitudinal. Reconhece-se que são de facto necessários estudos num contexto pragmático e realista; as estratégias terapêuticas e de diagnóstico devem ser avaliadas e as necessidades, perceções e preferências de pacientes e de prestadores de cuidados de

${ }^{1}$ Médica Assistente de Medicina Geral e Familiar. UCSP Vale de Cambra, ACeS Aveiro Norte.

${ }^{2}$ Médica Assistente de Medicina Geral e Familiar. USF São Miguel Arcanjo, ACeS Vale de Sousa Sul

${ }^{3}$ Médico Assistente de Medicina Geral e Familiar. USF La Salette, ACeS Aveiro Norte ${ }^{4}$ Médica Assistente de Medicina Geral e Familiar. USF Alfena, ACeS Maia/Valongo 
saúde devem ser também exploradas. ${ }^{1}$ É importante averiguar se estes recursos estão de facto a ser utilizados.

A investigação é essencial à afirmação e ao desenvolvimento da medicina geral e familiar (MGF) como disciplina científica. Em Portugal têm sido reconhecidas muitas dificuldades à investigação em MGF, mantendo-se a carreira académica e carreira clínica em caminhos geralmente separados, o que não facilita, provavelmente, o desenvolvimento da investigação nos serviços de saúde e clínicos. ${ }^{3}$

O programa de formação do internato médico da área profissional de MGF reconhece, contudo, a importância dos trabalhos de investigação na avaliação final dos candidatos. Segundo a Portaria n. ${ }^{\circ} 300 / 2009$, de 24 de março, ${ }^{4}$ mais recentemente atualizada na Portaria $n .{ }^{\circ} 45 / 2015$, de 20 de fevereiro, ${ }^{5}$ o programa de formação do internato médico da área profissional de especialização de MGF estabelece, como parte da avaliação final de internato, a apresentação de uma "Prova de discussão curricular - o curriculum vitae e que deve conter o resultado das avaliações formativas de desempenho e de conhecimentos obtidos ao longo do processo formativo". O colégio de Especialidade da Ordem dos Médicos, em conjunto com as Coordenações Regionais do Internato Médico de MGF, na sua reunião de 14 de setembro de 2012, homologou os critérios e, numa tentativa de uniformização, instituiu uma grelha de avaliação. ${ }^{6}$ Esta estabelece que $1 / 8$ da nota de quatro anos de trabalho do interno de MGF resulta diretamente do trabalho científico - projeto de intervenção, artigo de opinião, trabalhos de revisão, relatos de caso, trabalhos de revisão sistemática, trabalhos de garantia de qualidade e trabalhos de investigação - com valorização crescente, assumindo estes últimos uma importância maior.

No que diz respeito à caracterização da investigação realizada por médicos de família, a bibliografia existente é escassa. Num estudo realizado em 2004 na Alemanha, ${ }^{7}$ com o objetivo de avaliar a motivação dos médicos de família na participação em investigação nos CSP, verificou-se que $85,6 \%$ dos médicos incluídos no estudo revelaram uma atitude positiva face à realização de investigação. A principal motivação emanava da vontade de comprovar a qualidade dos cuidados através de estudos com maior evidência. Verificou-se, con- tudo, pouca experiência dos médicos na realização de estudos científicos, sendo as principais barreiras apontadas a sobrecarga de trabalho administrativo e clínico, o distanciamento entre a investigação teórica e o trabalho prático e o domínio desta área por outras especialidades. Num outro estudo realizado em $2012,{ }^{8}$ que incluiu 155 médicos de família especialistas a nível nacional, a investigação era uma das tarefas em que os médicos menos tempo despendiam ( $14 \%$ dos médicos em estudo, em média um minuto diário).

O presente estudo foi desenvolvido no Agrupamento de Centros de Saúde (ACeS) de Gondomar, criado em 2009, que engloba 14 unidades funcionais: uma Unidade de Cuidados de Saúde personalizados (UCSP), seis Unidades de Saúde Familiar (USF) modelo A e sete USF modelo B (90 médicos de família e 43 médicos internos de MGF). ${ }^{*}$ Com este estudo pretende-se quantificar a produção científica dos especialistas e internos de formação específica de MGF no ACeS Gondomar nos últimos dois anos, bem como identificar os motivos e fatores inerentes à sua realização.

\section{MÉTODOS}

Foi realizado um estudo descritivo e transversal no ACeS de Gondomar, que teve início em março de 2013 com a elaboração do protocolo de investigação. Este foi aprovado pelo Conselho Clínico do ACeS de Gondomar em janeiro de 2014 e pela Comissão de Ética da Administração Regional de Saúde do Norte (ARSN) em maio de 2014.

A população foi constituída por todos os médicos de família (orientadores e não orientadores) e internos de formação específica de MGF do ACeS de Gondomar, à data da realização do estudo. Foram excluídos todos os especialistas e internos ausentes do local de trabalho durante o período em estudo ou que se recusaram a participar, através do não preenchimento do questionário.

Em maio de 2014, os questionários foram entregues pelos autores a todas as unidades funcionais do ACeS de Gondomar. Os coordenadores de cada unidade, ou seus substitutos, voluntariamente aceitaram distribuir e recolher os questionários após o seu preenchimento,

*Informação recolhida junto do serviço de recursos humanos do ACeS de Gondomar em dezembro de 2014. 
que decorreu de maio até dezembro de 2014.

Cada questionário apresentava um texto introdutório em que se explicava o objetivo principal do estudo e se solicitava a participação voluntária, assegurando ainda a confidencialidade e anonimato no tratamento de todos os dados recolhidos. Após aplicação do questionário, este era selado num envelope e entregue ao coordenador de cada unidade. De seguida, foi atribuído a cada envelope, e de forma aleatória pelos investigadores, um número de identificação único. Os autores (internos do ACeS de Gondomar) foram incluídos no estudo.

As variáveis recolhidas e analisadas encontram-se caracterizadas no Quadro I.

Os autores consideraram um potencial viés de memória e, de forma a minimizá-lo, foi sugerido aos participantes o preenchimento do questionário com base no seu curriculum vitae relativo aos últimos dois anos de atividade.

Os dados recolhidos foram codificados e registados em base de dados informática, utilizando para o efeito os programas Microsoft Excel ${ }^{\circledR}$ e Statistical Package for Social Sciences $-S P S S^{\circledast}$ v22.0 para a análise estatística.

Tratando-se de um censo, os critérios de significância estatística e testes comparativos interamostras não se aplicaram. A comparação entre diferentes variáveis foi realizada de forma direta e apenas a significância clínica foi extrapolada.

A consulta e o trabalho de todos os dados foram realizados exclusivamente pelos autores do trabalho, garantindo-se, assim, pela obrigação ao sigilo médico, a confidencialidade dos dados obtidos para a realização do estudo.

As despesas de impressão dos questionários e as deslocações foram assumidas pelos autores.

\section{RESULTADOS}

Dos 133 médicos incluídos na população em estudo, 79 preencheram o questionário (taxa global de resposta de 59,4\%) - Quadro II. A taxa de resposta dos internos de MGF foi de $62,8 \%$ e de $57,8 \%$ para os especialistas de MGF, tendo-se verificado que a taxa de resposta foi superior nos médicos especialistas e orientadores de formação $(74,1 \%)$. Entre os internos de formação específica da especialidade, foram os do primeiro e quarto anos que obtiveram uma maior taxa de resposta.
A mediana de idades foi de 37 anos, sendo a idade mínima de 26 anos e a máxima de 61 anos. A população era constituída maioritariamente pelo género feminino (74\%). Relativamente ao local de trabalho, $57,0 \%$ trabalhavam numa USF modelo B, $41,7 \%$ numa USF Modelo A e 1,3\% numa UCSP.

Os participantes reportaram um total de 212 trabalhos científicos realizados nos dois anos prévios, o que correspondeu a uma média de 2,7 trabalhos por médico (Quadro III). O número médio de trabalhos científicos realizados pelos internos foi de seis trabalhos por interno, um valor superior ao que se verificou no grupo dos especialistas, tendo-se observado um aumento gradual ao longo dos primeiros três anos de internato (Quadro III).

Como se pode observar na Figura 1, a maioria dos trabalhos realizados foram revisões clássicas de literatura $(n=79)$, correspondendo a $37,3 \%$ do total de trabalhos realizados, seguidos dos relatos de caso com $23,1 \%$. A forma de divulgação através de comunicação oral em reuniões de serviço na unidade de saúde ou ACeS e através de pósteres foram as mais frequentemente observadas (28,8\% para cada uma, respetivamente), sendo a publicação de artigo a menos frequente $(1,4 \%)$ (Figura 2).

Em média, os participantes apontaram dois motivos para a realização de cada trabalho científico, sendo que os três motivos mais frequentemente reportados foram a melhoria do desempenho pessoal, enriquecimento do curriculum vitae e desenvolvimento da especialidade de MGF (Figura 3). A vontade do próprio e o orientador de formação foram os incentivos mais frequentes (Figura 4).

Dos 212 trabalhos científicos realizados, 3,8\% ( $n=8)$ foram premiados: três trabalhos de revisão clássica, dois relatos de caso, dois trabalhos de investigação e um trabalho de garantia de qualidade.

Cerca de $20,2 \%$ dos participantes estão satisfeitos com o número de trabalhos científicos realizados, $72,2 \%$ consideram que o número de trabalhos realizados não foi adequado à sua prática clínica e $7,6 \%$ não responderam à questão. Dentre as razões apontadas para tal, a falta de tempo e falta de horário dedicado à investigação foram as mais reportadas pelos participantes (42,0\% e 30,4\%, respetivamente) - Quadro III. Trinta e sete médicos $(46,8 \%)$ não realizaram qualquer 
QUADRO I. Caracterização das variáveis do questionário

\begin{tabular}{|c|c|}
\hline Nome da variável & Definição \\
\hline Número do questionário & Número de ordem do questionário atribuído pelo investigador \\
\hline Idade & $\begin{array}{l}\text { Registada em anos } \\
\text { Variável quantitativa contínua }\end{array}$ \\
\hline Género & $\begin{array}{l}\text { Variável qualitativa nominal (dicotómica) } \\
\text { Define-se por: } \\
\text { 1. "Masculino" } \\
\text { 2. "Feminino" }\end{array}$ \\
\hline Grau na carreira médica & $\begin{array}{l}\text { Grau académico/carreira que exerce na instituição à data do questionário } \\
\text { Variável qualitativa ordinal } \\
\text { Define-se por: } \\
\text { 1. "Médico especialista em MGF orientador de internato" } \\
\text { 2. "Médico especialista em MGF não orientador de internato" } \\
\text { 3. "Interno de MGF }-4 .^{\circ} \text { ano curricular" } \\
\text { 4. "Interno de MGF }-3 .^{\circ} \text { ano curricular" } \\
\text { 5. "Interno de MGF }-2 .^{\circ} \text { ano curricular" } \\
\text { 6. "Interno de MGF }-1 .^{\circ} \text { ano curricular" }\end{array}$ \\
\hline $\begin{array}{l}\text { Tipo de instituição do } \\
\text { ACES de Gondomar } \\
\text { onde exerce funções }\end{array}$ & $\begin{array}{l}\text { Variável qualitativa nominal } \\
\text { Define-se por: } \\
\text { 1. "UCSP" } \\
\text { 2. "USF modelo A" } \\
\text { 3. "USF modelo B" }\end{array}$ \\
\hline $\begin{array}{l}\text { Tipo de trabalho } \\
\text { científico }\end{array}$ & $\begin{array}{l}\text { Eventuais trabalhos desenvolvidos pelo especialista ou interno, nos últimos } 2 \text { anos, definidos com } \\
\text { base nos critérios da grelha de avaliação da ordem dos médicos } \\
\text { Variável qualitativa nominal } \\
\text { Define-se por: } \\
\text { 1. "Artigo de opinião" - Artigo publicado em que o autor dê a sua opinião sobre determinado } \\
\text { tema relevante para a profissão médica. } \\
\text { 2. "Relato de caso" - Descrição de um caso clínico com exploração do mesmo. } \\
\text { 3. "Trabalho de revisão clássica" - Pesquisa da bibliografia médica não estruturada sobre } \\
\text { determinado tema médico. } \\
\text { 4. "Trabalho de revisão sistemática" - Pesquisa da bibliografia médica estruturada, na qual foram } \\
\text { utilizados métodos claros e apropriados para identificar, avaliar, selecionar e resumir toda a } \\
\text { evidência de boa qualidade sobre determinado tema médico. } \\
\text { 5. "Trabalho de revisão baseada na evidência" - Trabalho de revisão sistemática não quantitativa, } \\
\text { com qualificação de afirmações segundo escalas taxonómicas em resposta a uma pergunta } \\
\text { objetiva inicial. } \\
\text { 6. "Trabalho de revisão de meta-análise" - Trabalho de revisão sistemática quantitativa com } \\
\text { implementação de bases estatísticas de sumarização da evidência. } \\
\text { 7. "Trabalho de garantia de qualidade" - Trabalhos cíclicos que visem a melhoria da prática médica. } \\
\text { 8. "Trabalho de investigação" - Trabalho em que variáveis de investigação sejam manipuladas ou } \\
\text { controladas pelos investigadores para a obtenção de uma resposta a uma pergunta científica. } \\
\text { 9. "Outro - Especificar". }\end{array}$ \\
\hline
\end{tabular}




\section{QUADRO I. Caracterização das variáveis do questionário (continuação)}

\begin{tabular}{|l|l} 
Nome da variável & Definição \\
\hline Forma de divulgação & Variável qualitativa nominal \\
& Define-se por: \\
1. "Comunicação em Poster" \\
2. "Comunicação oral em reunião da USF ou ACES" \\
3. "Comunicação oral em reunião do internato médico" \\
4. "Comunicação oral em congressos, jornadas ou encontros" \\
5. "Publicação em revistas médicas ou jornais sem avaliação interpares" \\
6. "Publicação em jornais e revistas médicas com avaliação interpares" \\
7. "Outro" - Especificar
\end{tabular}

Motivo para a realização Pertinência para a realização de cada trabalho científico, definidos pelos investigadores

do trabalho Variável qualitativa nominal definida por:

1. "Melhoria do desempenho pessoal"

2. "Melhoria do desempenho institucional"

3. "Desenvolvimento da MGF"

4. "De caráter obrigatório decorrente da atividade formativa"

5. "Enriquecimento do curriculum vitae"

6. "Compensação monetária"

7. "Outro - Especificar"

\begin{tabular}{|c|c|}
\hline Quem o incentivou & $\begin{array}{l}\text { Variável qualitativa nominal } \\
\text { Define-se por: } \\
\text { 1. "Coordenação do IFE de MGF" } \\
\text { 2. "ACES" } \\
\text { 3. "Equipa da Unidade de Saúde" } \\
\text { 4. "Grupo de investigação" } \\
\text { 5. "Orientador(a) de formação" } \\
\text { 6. "Próprio(a)" } \\
\text { 7. "Indústria farmacêutica" } \\
\text { 8. "Pós-graduação/Mestrado/Doutoramento" } \\
\text { 9. "Outro - Especificar" }\end{array}$ \\
\hline
\end{tabular}

\begin{tabular}{l|l}
\hline Trabalho premiado & $\begin{array}{l}\text { Premiado por instituição credível } \\
\text { Variável qualitativa nominal (dicotómica) } \\
\text { Define-se por: }\end{array}$
\end{tabular}
1. "Sim"

2. "Não"

Número de trabalhos realizados adequado
Se o investigador considera que o número de trabalhos realizados é adequado à sua prática clínica Variável qualitativa nominal (dicotómica)

Define-se por:
1. "Sim"
2. "Não"

\section{Variável qualitativa nominal}

Define-se por:

1. "Falta de tempo"

2. "Falta de horário dedicado à investigação"

3. "Falta de incentivos monetários"

4. "Falta de incentivos institucionais"

5. "Falta de colegas para integrar o grupo de investigação"

6. "Não tenho interesse na área"

7. "Outro - Especificar" 
trabalho nos últimos dois anos, tendo-se verificado que $81,1 \%$ destes consideram este valor não adequado à sua prática clínica - Figura 6 .

Assim, de entre os participantes que realizaram trabalhos científicos, destacam-se os internos de MGF $(57,1 \%)$, com mediana de 30 anos de idade, sendo que $78,6 \%$ eram do género feminino, com uma média de cinco trabalhos realizados e $54,8 \%$ pertenciam a USF modelo B.

\section{DISCUSSÃO}

Neste estudo, a taxa global de resposta $(59,4 \%)$ foi baixa. Os autores consideram que, apesar do fácil preenchimento, tal pode dever-se ao facto de o questionário ser extenso e do seu preenchimento estar dependente da memória do participante ou do curriculum vitae atualizado. Ainda, os autores optaram pela entrega dos questionários aos coordenadores de cada unidade, ou seus representantes, pelo que poderiam tê-los distribuído de outras formas suplementares para aumentar a taxa de resposta. A ausência na unidade de saúde durante os estágios hospitalares para os internos do segundo e terceiro anos, poderá também explicar a menor taxa de resposta observada nestes grupos (Quadro II). Tal facto poderia ter sido ultrapassado com o reforço, pelos autores junto dos coordenadores ou seus representantes, da importância do preenchimento dos questionários pelos internos em estágio, durante o período de ligação à unidade funcional e, também, com a entrega do questionário nas reuniões de núcleo de internos. Contudo, tal não explica a diferença nas taxas de resposta observadas entre os diferentes profissionais.

Uma outra limitação prende-se com a ausência de caracterização e comparação entre os médicos participantes e os que não responderam ao questionário, facto que se deve à confidencialidade inerente ao processo de preenchimento do mesmo.

O número médio de trabalhos científicos realizados por médico é muito variável entre os diferentes profissionais (Quadro III). A amostra estudada pode dividirse em dois grandes grupos: internos e especialistas de MGF. O valor médio no grupo dos internos parece ser devido à pressão para a produção de trabalhos científicos a que este grupo de profissionais se encontra su-

\section{QUADRO II. Caracterização dos participantes no estudo}

\begin{tabular}{lc} 
População & $\boldsymbol{n}$ \\
\hline Convidados & 133 \\
\hline Excluídos & $52 \dagger$ \\
& $2 \ddagger$ \\
\hline Total & 79
\end{tabular}

\begin{tabular}{l|c|c|c}
\hline Funções na instituição & $n$ & $\begin{array}{c}\text { Respostas } \\
(n)\end{array}$ & $\begin{array}{c}\text { Taxa de } \\
\text { resposta (\%) }\end{array}$ \\
\hline $\begin{array}{l}\text { Médico especialista em MGF } \\
\text { orientador de formação }\end{array}$ & 27 & 20 & $74,1 \%$ \\
\hline $\begin{array}{l}\text { Médico especialista em MGF } \\
\text { não orientador de formação }\end{array}$ & 63 & 32 & $50,8 \%$ \\
\hline Interno de MGF 4. ${ }^{\circ}$ ano & 12 & 8 & $66,7 \%$ \\
\hline Interno de MGF 3..$^{\circ}$ ano & 11 & 6 & $54,5 \%$ \\
\hline Interno de MGF 2..$^{\circ}$ ano & 12 & 5 & $41,7 \%$ \\
\hline Interno de MGF 1. ${ }^{\circ}$ ano & 8 & 8 & $100 \%$ \\
\hline Total & 133 & 79 & $59,4 \%$
\end{tabular}

Legenda - 'População excluída por não preenchimento do questionário.

‡Número de médicos excluídos por ausência do local de trabalho durante o períoo de estudo.

jeito. Para analisar estes resultados é fundamental considerar que a produção científica é muito importante para a avaliação curricular a que todos os internos de MGF são submetidos no final do seu internato, o que está de acordo com os resultados do presente estudo, onde se verifica um aumento gradual do número médio de trabalhos (nos dois anos prévios) com o ano de internato (Quadro III). A ligeira diminuição que se verifica no último ano do internato é, provavelmente, devida à necessidade de realização de atividades obrigatórias (estudo da lista do orientador de formação e processo de escrita do curriculum vitae), aliadas à atividade assistencial na unidade de saúde.

No que diz respeito ao tipo de trabalhos científicos realizados, $60,4 \%$ foram trabalhos de revisão clássica e relatos de caso (Figura 1). Presume-se que tal aconteça por serem trabalhos de uma execução aparentemente menos complexa e mais rápida em comparação com meta-análises, revisões sistemáticas e trabalhos de garantia de qualidade.

De igual forma, os processos de divulgação mais escolhidos não foram os que apresentam maior impacto 


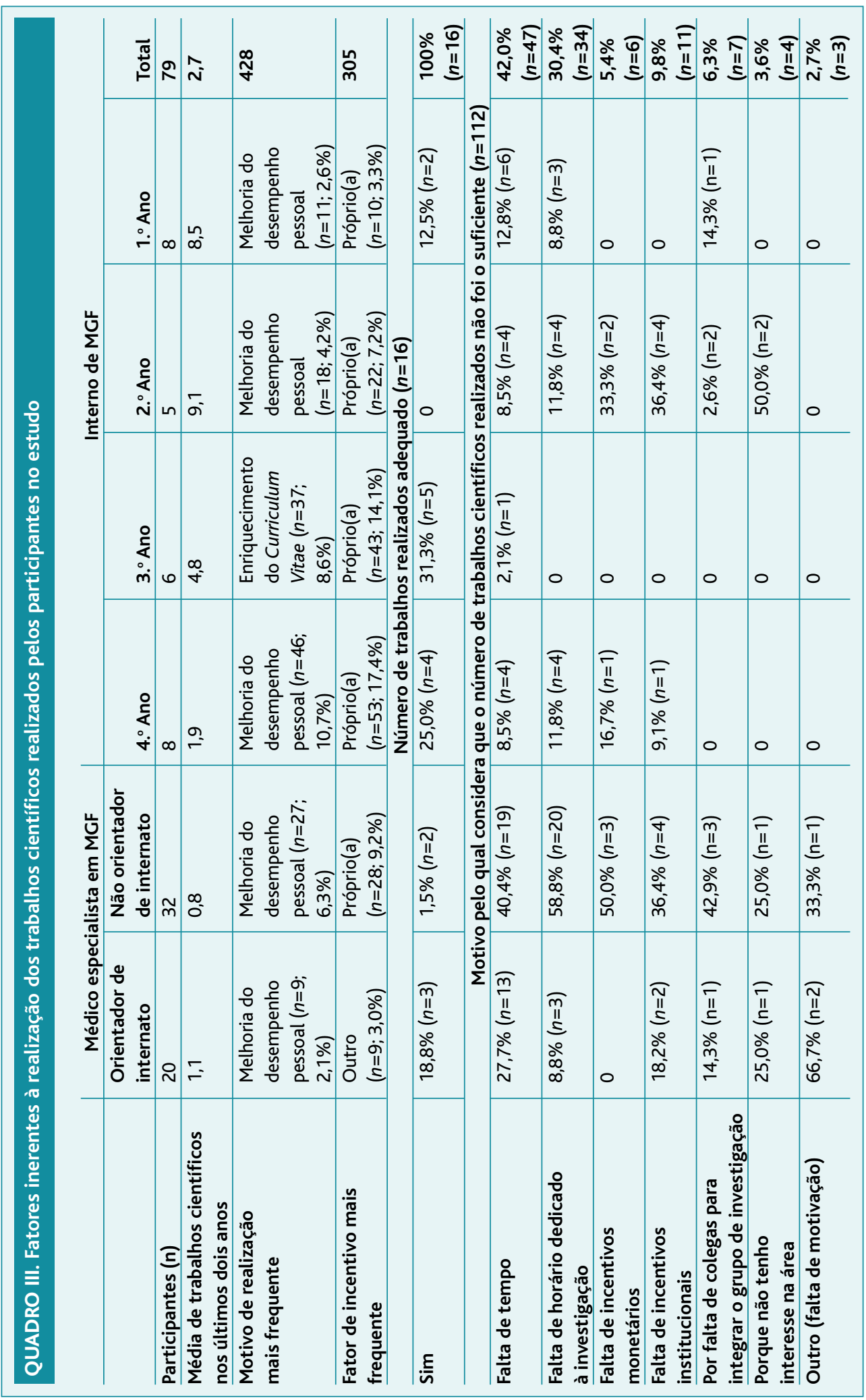


na comunidade médica, mas as formas mais acessíveis de expor um trabalho realizado (Figura 2), sendo a publicação de artigos (com ou sem revisão interpares) praticamente inexistente na amostra. Tal deve-se, provavelmente, à escassez de incentivos para publicação que, na maioria das vezes, é um processo moroso. Como referido anteriormente, os internos de MGF são o grupo profissional que executa um maior número de trabalhos científicos. Não obstante, são também o grupo profissional com a remuneração inferior (relativamente aos estudados). Assim sendo, as taxas de publicação exigidas por certas revistas podem ser uma dificuldade adicional para a publicação.

Relativamente aos motivos e incentivos para a realização dos trabalhos científicos, as regras de preenchimento do questionário não faziam referência à ordem de importância (crescente ou não) dos mesmos, pelo que é possível apresentar apenas os motivos e incentivos mais relevantes de acordo com a frequência com que foram assinalados. Esta é uma das limitações do presente estudo.

Quanto aos motivos para a realização de cada trabalho científico, tendo em conta a implicação avaliação dos internos de MGF e dado o congelamento da progressão nas carreiras médicas, compreende-se facilmente que a razão «enriquecimento do CV» se apresente como um dos motivos mais frequentemente apontados, particularmente pelos internos.

As entidades mais próximas do médico (orientador de formação,

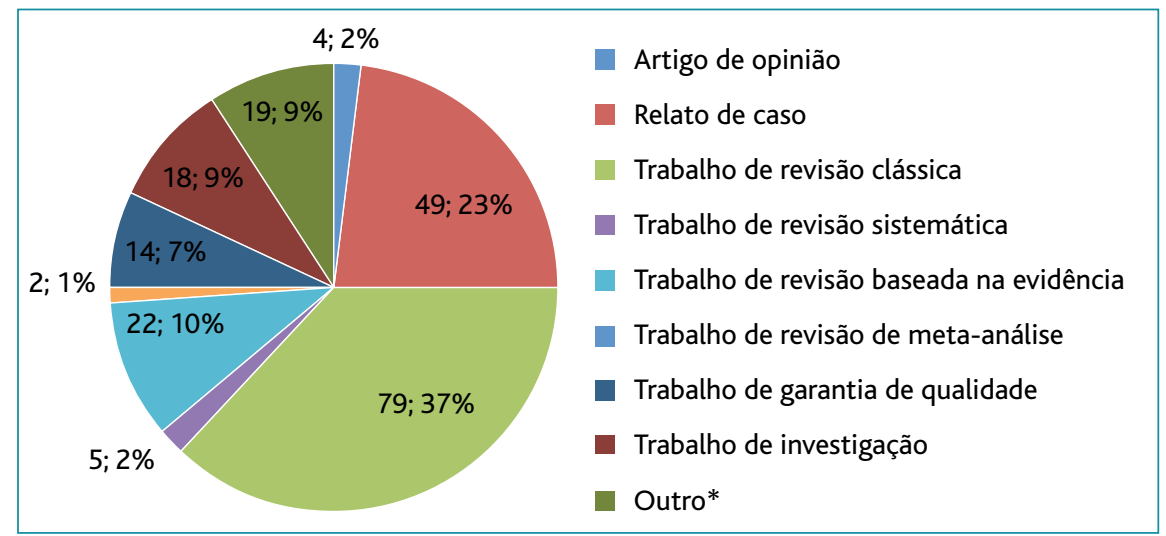

Figura 1. Número e tipo de trabalhos científicos realizados $(n=212)$.

Legenda - *Sessões de educação para a saúde, normas de orientação clínica, workshop.

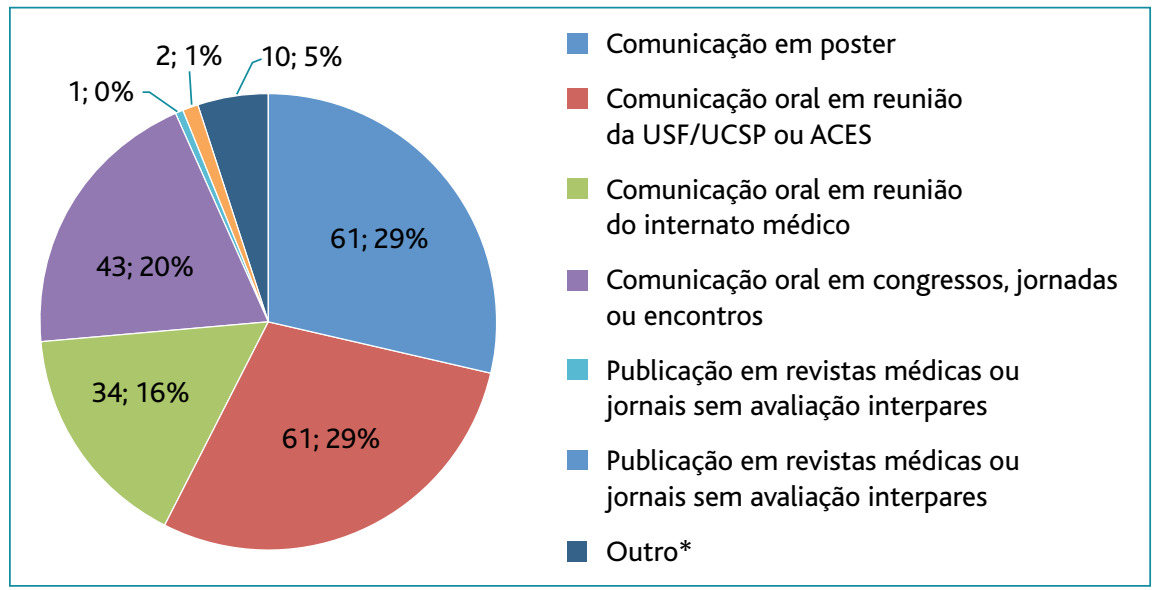

Figura 2. Número de trabalhos científicos realizados e forma de divulgação $(n=212)$. Legenda - *Sítio da internet, sessões de educação para a saúde, comunicação oral em reunião de serviço hospitalar.

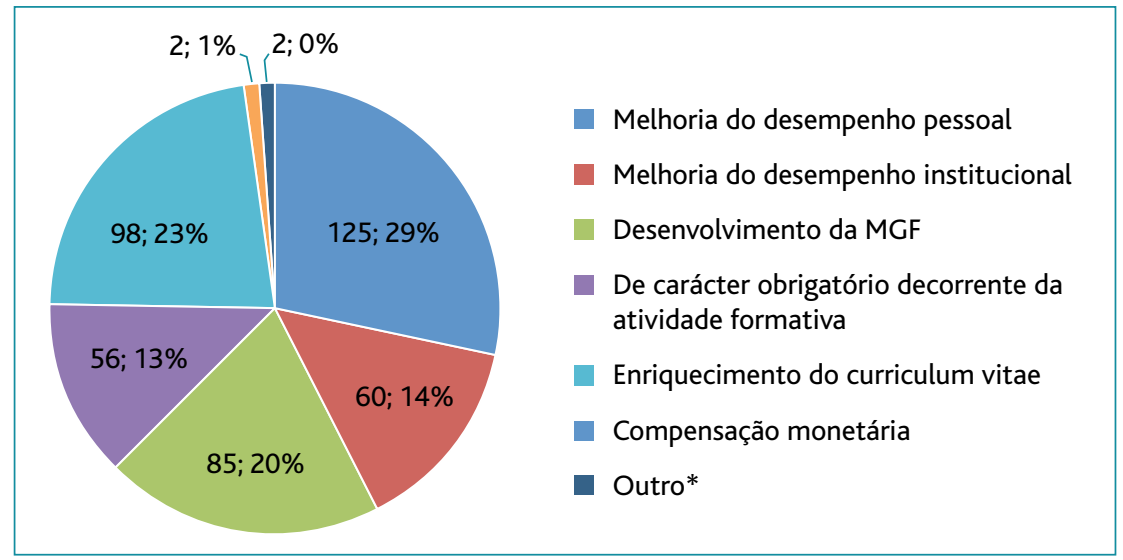

Figura 3. Razões para a realização dos 212 trabalhos científicos $(n=428)$.

Legenda - *Não especificado. Nota: os participantes podiam selecionar no máximo três opções de resposta. 


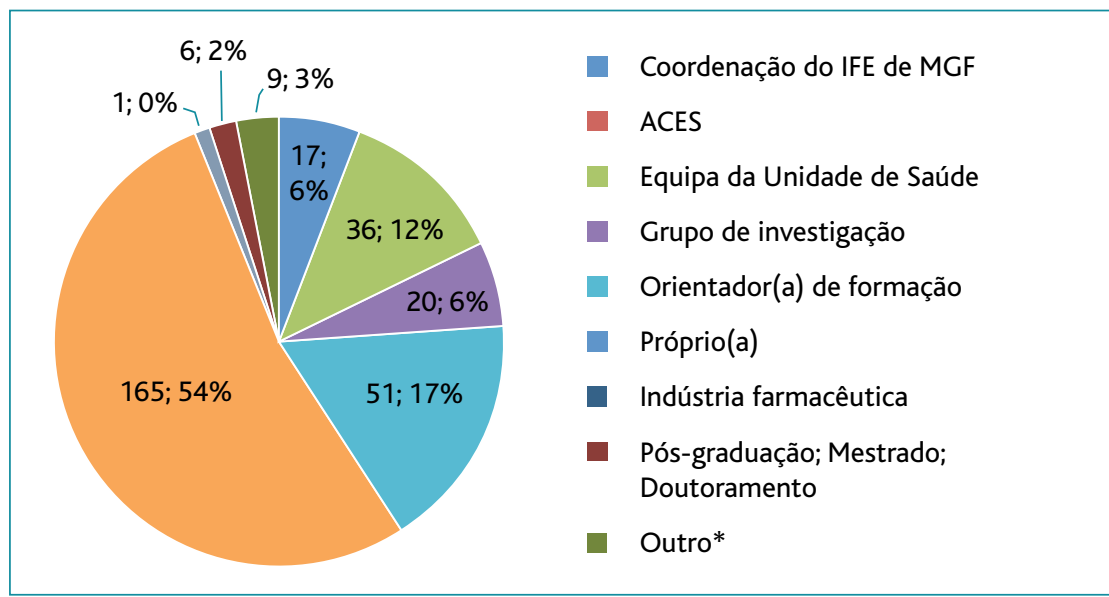

Figura 4. Fatores que motivaram a realização dos 212 trabalhos científicos $(n=305)$. Legenda - *Não especificado. Nota: os participantes podiam selecionar no máximo três opções de resposta.

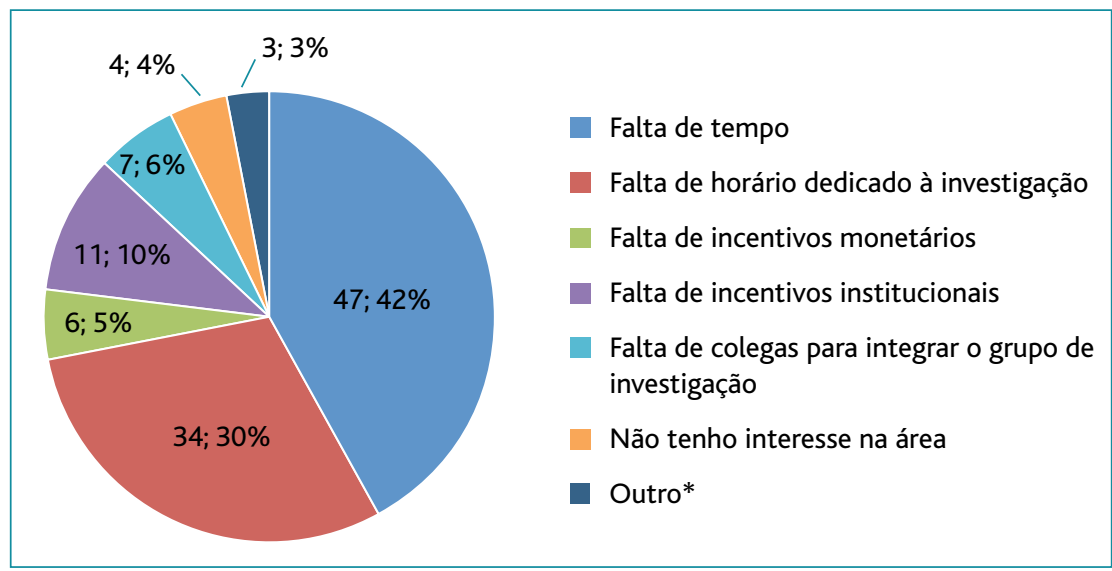

Figura 5. Razões reportadas pelos participantes que consideram o número de trabalhos científicos realizados não adequado à sua prática clínica $(n=112)$.

Legenda - *Falta de motivação. Nota: os participantes podiam selecionar no máximo três opções de resposta.

unidade de saúde) foram as principais fontes de motivação para a realização de trabalhos científicos. A manutenção de ligações fortes e saudáveis entre o médico de família e as entidades que lhe são próximas poderá contribuir para o desenvolvimento de mais e melhor investigação em MGF.

A maioria dos participantes $(72,2 \% ; n=57)$ considerou que o número de trabalhos científicos realizados não se adequava à sua prática clínica. As razões identificadas pelos médicos para estes resultados (falta de tempo, falta de horário dedicado à investigação e falta de apoio institucional) poderão ser entendidas como áreas de intervenção prioritária. É importante salientar que um número considerável de médicos $(n=37)$ referiu não ter realizado nenhum trabalho científico nos últimos dois anos - Figura 6. Observamos ainda que a satisfação com o número de trabalhos realizados é muito variável, o que poderá estar relacionado com diferentes níveis de exigência individual.

Com o presente estudo pretendeu-se dar uma visão geral do perfil dos principais investigadores no ACeS de Gondomar e dos fatores inerentes à realização de trabalhos científicos pelos médicos de MGF (especialistas e internos). Os resultados sugerem que, no ACeS de Gondomar, a maioria dos médicos está insatisfeita com o tempo e com a falta de apoio institucional para a realização de trabalhos científicos e que a maioria considera insuficiente $o$ trabalho realizado. Os internos de MGF, seja por motivação pessoal ou por enriquecimento do $\mathrm{CV}$, são os que realizam a maioria do trabalho científico. Apesar de a amostra não ser representativa da população de médicos de MGF do ACeS de Gondomar (para tal seria necessário uma amostra de 99 participantes), os autores consideram que este estudo é uma mais-valia por ser uma primeira abordagem a uma questão muito mais complexa: a inexistência de infraestruturas, recursos e investimentos para o desenvolvimento de investigação na MGF, prática e adequada à realidade. ${ }^{3}$ Será de esperar, contudo, que quem participou esteja, à partida, também mais sensibilizado para o tema e, por isso, tenha mais trabalhos realizados ou, em última instância, mais cientes da sua importância.

É importante salientar alguns aspetos que devem ser considerados em futuras investigações sobre esta temática, nomeadamente na aplicação do questionário (tornando o seu preenchimento mais simples e menos 


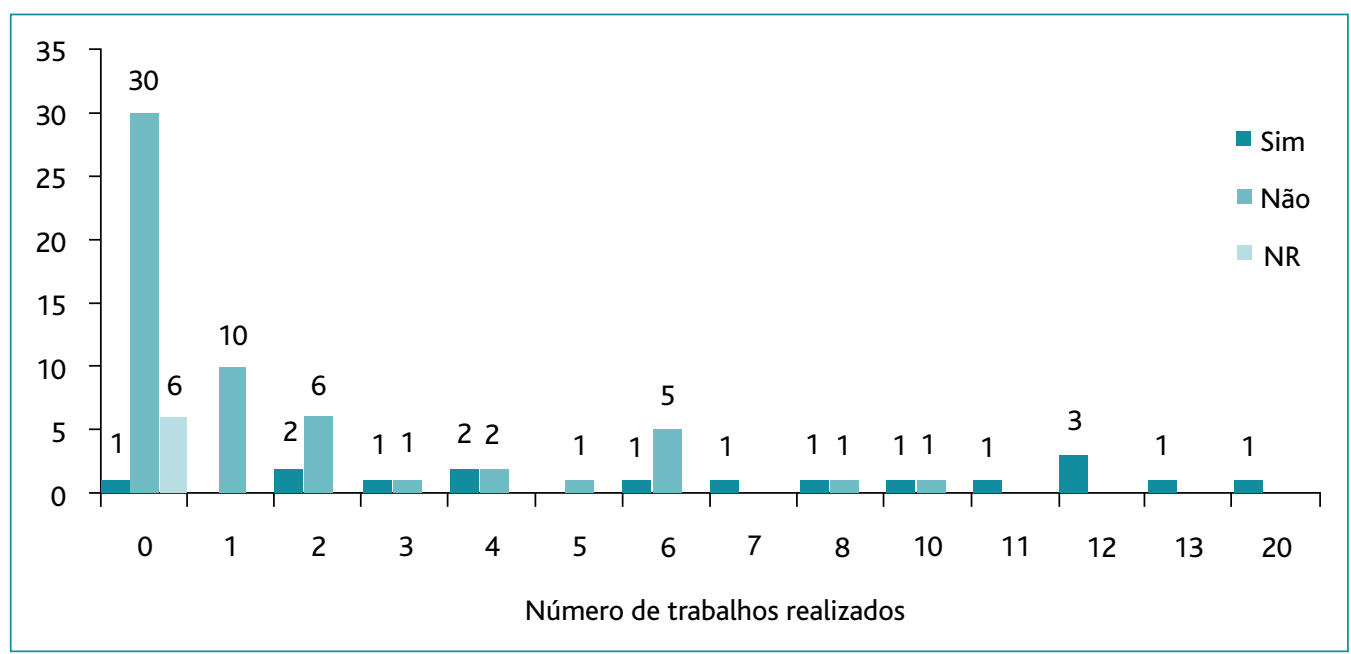

Figura 6. Relação da satisfação e o número de trabalhos realizados.

Legenda - Sim: número de trabalhos realizado adequado à prática clínica. Não: número de trabalhos realizados não adequado à prática clínica. NR: não responde

moroso) e respetiva distribuição (através da sua aplicação via Internet ou em reuniões de internato).

A dinâmica da especialidade de MGF está em evolução, assim como os CSP, mas há ainda um longo caminho a percorrer no que diz respeito às condições de formação das unidades de saúde, bem como dos médicos e orientadores de formação. É necessário criar o conceito de unidades de saúde formativas com plano próprio, de modo a proporcionar e manter qualidade na formação e que contemple a realização de trabalhos científicos.

\section{AGRADECIMENTOS}

Os autores gostariam de agradecer a colaboração do ACeS de Gondomar e todos os profissionais que aceitaram participar neste estudo.

\section{REFERÊNCIAS BIBLIOGRÁFICAS}

1. Hummers-Pradier E, Beyer M, Chevallier P, Eilat-Tsanani S, Lionis C, Peremans $L$, et al. Research agenda for general practice/family medicine and primary health care in Europe [Internet]. Maastricht: European General Practice Research Network; 2009. Available from: http://www. egprn.org/files/userfiles/file/research_agenda_for_general_practice_family_medicine.pdf

2. Is primary-care research a lost cause? Lancet. 2003;361(9362):977.

3. Braga R. A necessidade de nos afirmarmos na investigação. Rev Port Clin Geral. 2011;27(3):257-8.

4. Portaria n. ${ }^{3}$ 300/2009, de 24 de março. Diário da República. 1. Série(58).
5. Portaria n. 45/2015, de 20 de fevereiro. Diário da República. 1. ${ }^{\text {a Sé- }}$ rie(36).

6. Colégio da Especialidade de Medicina Geral e Familiar. Avaliação final do internato médico de medicina geral e familiar: aprovado pelo CNE em 14.09.2012. Lisboa: Ordem dos Médicos; 2012 [cited 2015 Feb 24]. Available from: www.ordemdosmedicos.pt

7. Rosemann T, Szecsenyi J. General practitioners' attitudes towards research in primary care: qualitative results of a cross sectional study. BMC Fam Pract. 2004;5(1):31.

8. Granja M, Ponte C, Cavadas LF. What keeps family physicians busy in Portugal? A multicentre observational study of work other than direct patient contacts. BMJ Open. 2014;4:e005026.

\section{CONFLITOS DE INTERESSE}

Os autores declaram que não têm nenhum interesse financeiro, nem receberam nenhuma assistência editorial de qualquer organização ou entidade com interesse financeiro no tema do manuscrito.

\section{COMISSÃO DE ÉTICA}

Estudo realizado após parecer favorável da Comissão de Ética da ARS Norte.

\section{ENDEREÇO PARA CORRESPONDÊNCIA}

Patrícia Fernandes

$A v^{a}$ General Humberto Delgado, $n^{\circ} 794-2^{\circ} \mathrm{dt}^{\circ}$

4420-155 S. Cosme - Gondomar

E-mail: patricia.fernandes99@gmail.com

Recebido em 24-07-2015

Aceite para publicação em 22-08-2016 


\section{ABSTRACT}

FACTORS RELATED TO THE PERFORMANCE OF RESEARCH IN THE GONDOMAR GROUP OF HEALTH CENTRES: A CROSS-SECTIONAL STUDY

Objectives: Research is essential for development of general practice as a scientific discipline. In Portugal, there are many difficulties in performing research in primary health care. This study was conducted to quantify the scientific output of family doctors and trainees in primary care units in Gondomar, to understand their motivation for doing research, and to assess the factors related to successful completion of research.

Type of study: Cross-sectional study.

Location: Gondomar Group of Health Centres, Portugal.

Population: Family medicine specialists and trainees.

Methods: We performed a census among the 133 general practitioners in the Gondomar Group of Health Centres using an original questionnaire. The variables studied were the number and of studies performed by the participants over a two-year period. We also asked participants about their satisfaction with the amount of research done and the factors that supported the performance of research.

Results: We received 79 completed questionnaires for a response rate of $59.4 \%$. There were 212 studies reported for a mean of 2.68 studies per doctor. Trainees were involved in a higher number of studies than specialists, with a mean of 6 studies per trainee. About half the doctors (46.8\%) were not involved in any studies during the study period and over half of the participants (57\%) were not satisfied with the number of studies done. Lack of time in general and time for research in particular were the main reasons given for not conducting research.

Conclusion: General practice in Portugal is evolving but there is still a long way to go in promoting high quality research in primary health care centres.

Keywords: General practitioners; Research; Primary care. 